\title{
A new method of brain stimulation at ultra-high frequency
}

\author{
Yousef Jamali1*, Mohammad Jamali2,3, Mehdi Golshani²,3 \\ 1) Department of Applied Mathematics, School of Mathematical Sciences, Tarbiat Modares University, Tehran, Iran \\ 2) Department of Physics, Sharif University of Technology, Tehran, Iran \\ 3) School of Physics, Institute for Research in Fundamental Sciences (IPM), Tehran, Iran \\ * Correspondence: y.jamali@ipm.ir
}

\section{Summary}

Nerve stimulation via micro-electrode implants is one of the neurostimulation approaches which is used frequently in the medical treatment of some brain disorders, neural prosthetics, brainmachine interfaces and also in the cyborg. In this method, the electrical stimulation signal can be categorized by the frequency band: low frequency, high frequency, and ultra-high frequency. The stimulation should be less destructive, more smooth, and controllable. In this article, we present a brief description of the mechanism underlying the ultra-high frequency stimulation. In the flowing, from an informatics perspective, we propose a state-of-the-art, low destructive, and highly efficient stimulation method at the low amplitude ultra-high frequency signal. In this method, we have tried to reduce the adaptation of the nerve system by modulating the stimulation signal via a low frequency rectangular random wave. By this method, we could reach the "almost zero discharge" with minimum destructive effect in the experimental test on the fish nervous system.

\section{Keywords}

Deep Brain Stimulation; Electrical Stimulation; Ultra-High Frequency Stimulation; Fish Locomotion Control. 


\section{Introduction}

The purpose of electrical nerve stimulation (neurostimulation), that includes implanting electrodes in the nerve system and applying an electric field to it, is pushing neurons to fire. Many factors, such as the shape and material of electrodes [1-11], implanted area (including its symmetry and asymmetry, etc. [12-14]), the property of signal (such as: shape, amplitude, and frequency) [1, 15$21]$ and the type of stimulation (i.e. constant voltage, constant current or constant charge $[1,5,15$, $22,23])$ can be affected the quality of stimulation.

The adverse effects of direct current stimulation (DCS) or long-pulsed current stimulation (PCS), such as tissue and electrode damaging [1, 3], making them inappropriate for nervous system stimulates. Hence caution should be exercised when using these methods in some sensitive applications, such as the deep brain stimulation (DBS). Of course, this method is still useful for the blocking of the nerve system $[24,25]$.

In the neurostimulation, applied frequency bands (range, spectrum) could be categorized into a lower frequency $(<100 \mathrm{~Hz})[8,24-34]$ higher frequency $(100-1000 \mathrm{~Hz})[4,7,9,11,35]$ and ultrahigh frequency $(>500 \mathrm{~Hz}$ ) [36-38]. Low-frequency (The lower band) stimulation is used in most of the works since the physiological frequency of neurons typically is less than $100 \mathrm{~Hz}$ [37] (though it could reach up to $700 \mathrm{~Hz}$ in some cases [36, 39]). This physiological restriction of frequency is related to three facts;first: the existence of a refractory period following each action potential which is in the order of milliseconds; second: limitation in the speed of synaptic action, and third; temporary depletion of synaptic vesicles at a persistent high $(>100 \mathrm{~Hz})$ frequency stimulation (synaptic fatigue) [36, 37, 40-43].

Over recent years, the use of high-frequency bipolar stimulation (HFBS) has been extensively increased. This tendency is due to some factors such as: reduction of adverse physiological and electrochemical effects at HFBS [1, 3, 4, 16, 44-47], the experimental confirmation of the possibility of nerve stimulation at high frequency $(\sim \mathrm{kHz})$ [37] (perhaps, because of the accumulation effect [37, 48, 49]) and variety of effective parameters at the HFBS [37]. Although the experimental results indicate the successfulness of this method $[37,50]$ there is no global, onesize-fits-all model to explain the mechanism of stimulation in this frequency range[36, 37]. Different models have been used in order to explain some aspects of the behaviors. 
Ultra high frequency has been mainly used for three cases: nerve conduction blocking, radiofrequency nerve ablation (and heating), and nerve stimulation. In the nerve conduction blocking, which used for example in chronic pain treatment [36, 37, 51-54], stimulation signal in the $\mathrm{kHz}$-frequency $(1-100 \mathrm{KHz})$ is continuously applied to the tissue. This stimulation prevents signal transmission through the nerve, by blocking axons conduction [36].

The radiofrequency $(>500 \mathrm{KHz})$ nerve ablation, by heating up a small area of nerve tissue, eliminates the nerve and cancer tissue or relieves the pain (because of decrease in the nervous system's activity at high temperatures)[3]. In the nerve stimulation, which is mainly used in electrotherapy [37] and cyborg [7, 55], the ultra-high frequency is modulated by a low-frequency carrier signal $(50-100 \mathrm{~Hz})$. A variety of modulation techniques, such as interference of multi different high-frequencies or using carrier square pulse, have been developed [37].

In the last application (nerve stimulation), after applying an ultra-high frequency stimulation, a brief onset response, followed by an exponential decay of the firing rate, have been observed experimentally and studied theoretically $[36,56]$.

To prevent this decay, the signal could be switched off and on periodically. The off state gives enough time for the nerve cells to recover and prepare them for the next stimulation. Hence the switching frequency's choice is based on the recovery time and is in the order of natural frequency of firing (Figure 1).

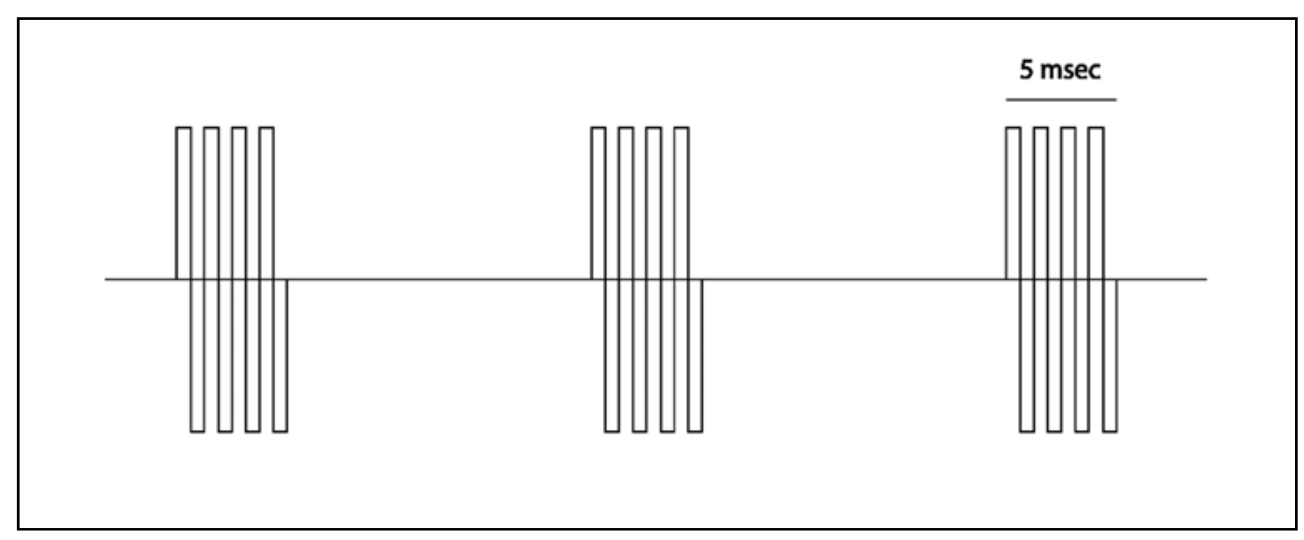

Figure 1. a stimulation signal at an ultra-high frequency that switches on \& off at low frequency. 
In this article, after the investigation of high-frequency stimulation mechanism, our aim is to find the optimal stimulation (which is more effective with less signal strength). We shall show that neuronal ultra-high frequency stimulation, when modulated on with a random time-varying lowfrequency carrier, will result in an optimal stimulation with lower adverse effects. To confirm our hypothesis, some fish brains were stimulated electrically in vivo, using the aforementioned method and we compared it with other methods in terms of its charge injection (charge and discharge amplitude) per cycle and fish locomotion respond.

\section{Ultra-high frequency electrical stimulation}

As we outlined above, stimulation at an ultra-high frequency $(3-500 \mathrm{KHz})$ and with an intensity which would not trigger the nerve blocking [36], leads to the initiation of neuronal firing. However, the firing rate decays exponentially to zero with a time constant of multi-seconds [39, 57-61]. Hence applying this stimulation constantly, if doesn't cause blocking ( nerve conduction block or neurotransmitter depletion block [36, 37, 40-43]) causes an onset respond proportional to the intensity of the excitation current. However, this response is transient and will not last, as shown schematically in Figure 2.

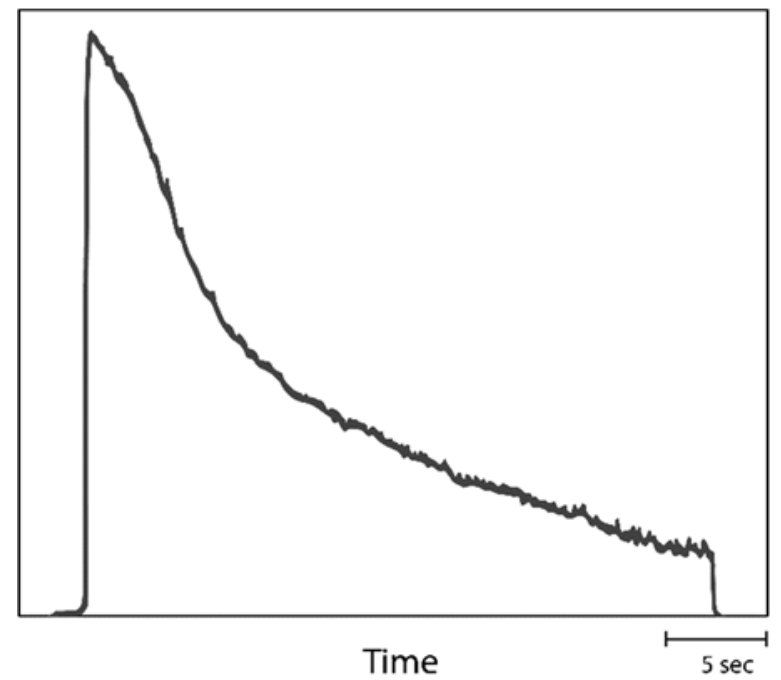

Figurer. schematic pattern of the onset response of a neuron to ultra-high frequency stimulation with low intensity. The picture is adapted from [36]

After stopping the stimulation (i.e. turning off the neurostimulator), the organism starts to recover [The recovery time depends on the stimulation parameters, such as frequency, amplitude, and shape, as will be discussed in details in the following.] and returns to its normal mode. Therefore, 
by alternately connecting and disconnecting an appropriate very high-frequency stimulation by means of enough low-frequency signal, it is possible to achieve a long-lasting and steady nerve system response. There are some common methods which use this approach:

1. Using a superposition current [37, 62-68]. This method, as is shown in Figure 3a, involves the application of two currents (usually $\sim 2.5 \mathrm{KHz}$ sinusoidal signal) at slightly different frequencies $(\sim 50 \mathrm{~Hz})$. These two waves interfere with each other and create a modulated lowfrequency signal of $\sim 50 \mathrm{~Hz}$. The wavelets will repeat at regular intervals.

2. Using a modular square current [37, 66-70]. This involves switching on and off of a highfrequency square/sinusoidal signal $(1-100 \mathrm{KHz})$. The switching is driven by a square lowfrequency signal $(50 \mathrm{~Hz})$, with an adjustable duty [37] (Figure 3b). This type of stimulation, due to the duty control, is less destructive during a short stimulation and is more efficient.

3. Using a high-frequency stimulation with non-zero offset. In the ref. [71] a burst sinusoidal high frequency, with a non-zero offset, was used for stimulation (Figure 3c). In addition, in the ref. [72], authors suggested a voltage control stimulus which is switched on and off repeatedly, with a high frequency (upto100kHz) duty cycled signal (non-zero offset).

4. Using a low-frequency rectangular waveform stimulation with low Duty-cycle (Figure 3d). This type of stimulation is classically placed into the current pulse (PC) stimulation category. The fact of the existence of high-frequency components in its Fourier spectrum makes it belong to the high-frequency stimulation in order of $1 / \mathrm{T}$ which $\mathrm{T}$ is the pulse duration. Especially, in the constant voltage stimulation, due to the capacitive property of environment and electrodes, the shape of current is a bipolar periodic wave with the same pulse duration (Figure 4). Recently this method has been widely used in the DBS [16, 47, 73-79]. Because of low Dutycycle, it, too, would have little adverse electrochemical effects [1, 3, 47]. 
a)

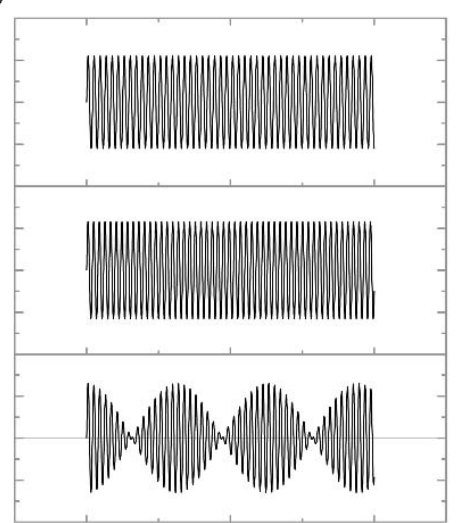

c)

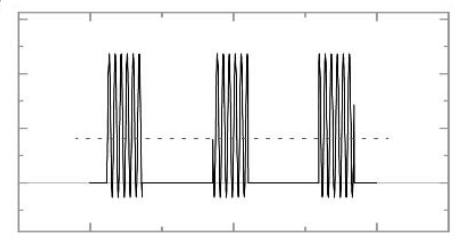

b)

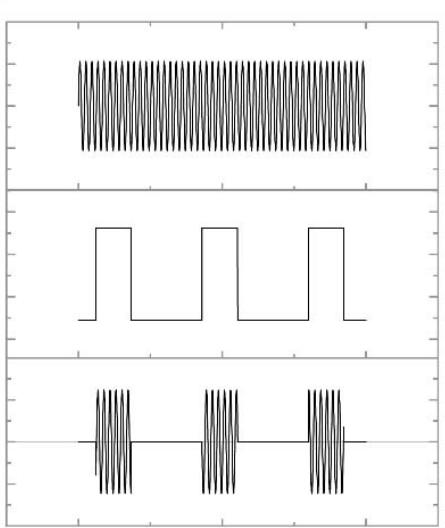

d)

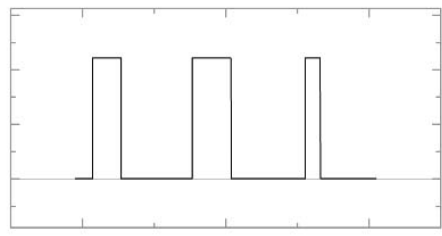

Figurer : By alternately connecting and disconnecting an appropriate very high-frequency stimulation by means of enough lowfrequency signal, it is possible to achieve a long-lasting and steady nerve system response. There are some common methods which use this approach: a) Using a superposition current; b) Using a modular square current; c) Using a high-frequency stimulation with non-zero offset; d) Using a low-frequency rectangular waveform stimulation with low Duty-cycle.

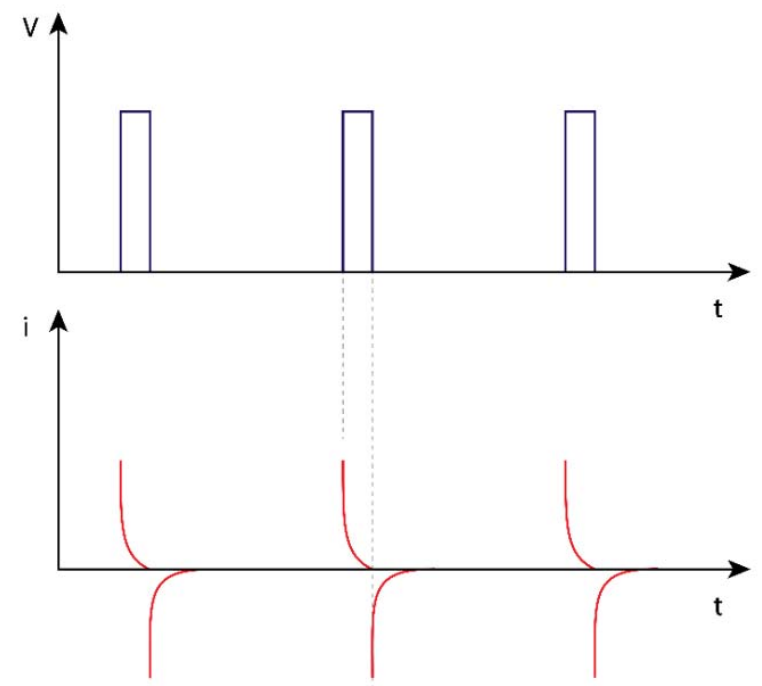

Figure 4: In the constant voltage stimulation (top figure), due to the capacitive property of environment and electrodes, the shape of current is a bipolar periodic wave with zero charge balance (down figure). 
Using bipolar alternative stimulation, with zero charge balance, could offer stimulation with less adverse effects. Table 1 shows some examples of applied stimulation, with the zero charge balance.

\section{Biophysical mechanism of high-frequency stimulation effects}

The reaction of the nerve system to the stimulation methods outlined above consists of two stages. The first stage is an initial respond of neurons to the ultra-high frequency stimulation, and the second one is the recovery and returns to the resting state. Both stages are discussed in more detail below.

\section{The first stage:}

As the transmembrane field is increased, due to the right external electric field, the opening probability of a given voltage-gated sodium channel is raised. Consequently, the number of open channels is increased. Due to the existence of an asymmetry in the closing and opening dynamics of channels, for an open channel, by removing the external field or even applying the inverse one, these channels will not be closed (by the same opening mechanism). Hence if the field amplitude and the frequency are high enough $[51,52]$ so that the summation phenomena occur $[37,48,49]$, after certain periods, the induced membrane fields reach the threshold and initiate an action potential, (Figure 5). This onset response, both in-vitro [37, 48, 51, 52] and in-silico [53, 54, 80, 81] have been well characterized. It's worth noting that according to the nerve membrane time constant $(\tau=R C \sim 10 \mathrm{~ms})$, the stimulation frequency must be more than $\frac{1}{\tau}$, which is in the high frequency range.

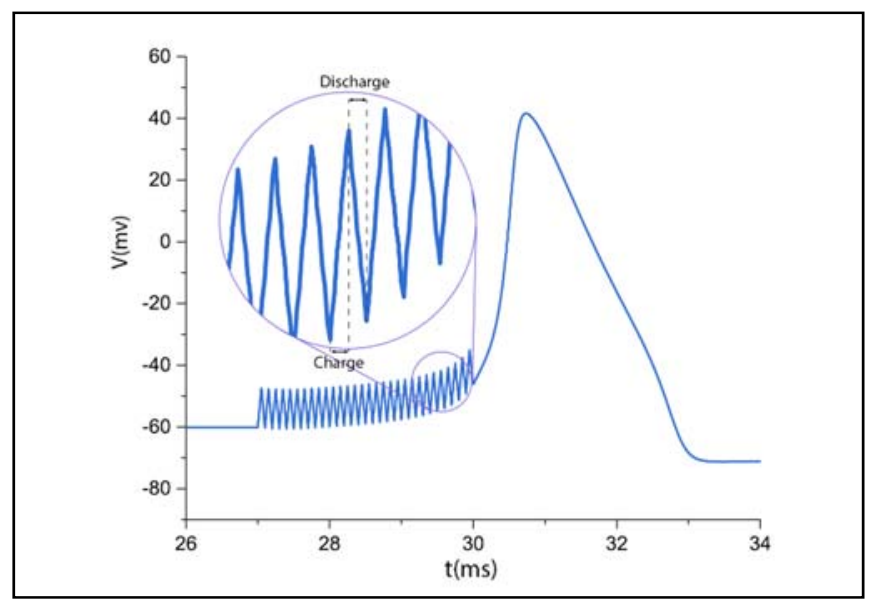

Figure 5. Simulation result of neuron responds and variation of membrane potential under high-frequency stimulation based on the H\&H dynamics model [our unpublished simulation result]. 
In addition to the axon's characteristics, like diameter and length, the intensity of neuron's firing depends on the frequency and the amplitude of applied field [36, 39]. In principle, increasing the frequency of stimulation increases stimulation or, in other words, results in more efficient stimulation (efficient stimulation means reducing the threshold of the applied field intensity of stimulation neurons or more stimulation in a determined intensity). The main reasons for this is, on the one hand, the asymmetry in the dynamics of channel activation based on the H\&H equations (Figure 5 shows the simulation result of neuron dynamics under H\&H dynamics), and on the other hand, an increase in the effective field (the transmembrane fields resulting from the applied filed) which causes the rise of opening probability of sodium channels [our unpublished simulation result]. This will be discussed in the next session.

\section{The effect of an induced electric filed on raising the probability of firing}

In the high-frequency stimulation, if the field amplitude is decreased (due to the reduction in the opening probability of sodium channels), while the closing rate is constant and is independent of field amplitude, then sodium current decreases. Hence, it can result in a lack of the summation process [1]. In other words, the effective time and the amplitude of stimulation should be large enough, so that the opening rate of sodium ion channels overcome their closing rate. In this situation, the system leaves its resting state and goes to the firing mode. Nevertheless, in the low amplitude situation, although the field itself does not directly cause neurons to fire, it does change and even elevates the transmembrane potential. This elevation, in turn, results in increasing the neuron sensitivity to other external stimulations. Thus, firing likelihood goes up, compared to the normal situation.

As we know, neurons have a baseline firing rate in-vivo, due to different parameters, such as random synapsis current and/or environmental noise. Hence the above mentioned increased sensitivity results in the elevation of baseline firing rate. In other words, even at low amplitudes, for which no direct stimulation can occur, the baseline rate of neurons will increase, because of their higher sensitivity.

Hence, applying a low amplitude field could be equal to an increase in the response of neurons to the environmental stimulation, especially the environmental noise. As a result, the baseline firing rate of a neuron under such stimulation is higher compared to other neurons. In the following 
section, we shall outline how increasing the duration of an applied field, regardless of the summation property, could increase the probability of stimulation, especially at symmetric high frequencies.

\section{Increasing the effective time via ultra-high frequency stimulation.}

Just after applying electrical stimulation, ions around the electrodes move and a plane of charge, at the surface of the metal electrode, is opposed by a plane of opposite charge, as "counterions", in the electrolyte. This double layer capacitor around the electrodes [1, 3, 82-84], as well as shielding of electrodes by polar molecules, such as water and polar proteins, results in induced electric fields decaying exponentially to zero with a time constant of 10 microseconds $[1,3]$. Hence neurons around the electrode sense the stimulation field only in the first few microseconds, which we term as effective time [In fact, what we explain here is just a non-faradaic current. There is two types of currents, Capacitive current, which is also called "non-faradaic" or "double-layer" current, does not involve any chemical reactions. It only causes accumulation (or removal) of electrical charges on (or from) the electrode and in the electrolyte solution near the electrode. This type of current is reversible and nondestructive. On the other hand, the faradaic current is the current generated by the reduction or oxidation of some chemical substance at an electrode. The net faradaic current is the algebraic sum of all the faradaic currents flowing through an electrode. This type of current is destructive and we prefer reducing it as much as possible. At low amplitude electric fields, the faradaic current is negligible]. When the polarity of the applied voltage source is reversed, the direction of current is reversed, the charge redistribution is reversed, and the charge that was injected from the electrode into the electrolyte and stored by the capacitor may be recovered. As a result, by increasing the frequency of the charge balanced stimulation, it is possible to reach a longer total effective time in the defined period of time.

In both capacitive [1-3] and voltage-controlled (potentiostatic) stimulation methods [1], which are in the electrochemical reversibility range $[85,86]$ [Several experimentalists have made the choice of using voltage-controlled stimulations to remain within the reversible range of the electrodes (Joucla and Yvert, 2012).], the implementation of a square wave stimulation provides a decreasing exponential current in the tissue and electrode (the faradaic current can be removed by adding an external serial capacitor to the circuit). Hence in accordance with quasi-static or quasi-stationary equations $[18,19,87-90]$, an intense current, at the initial moments of stimulation $(\sim 10 \mu s)$, results in an induced electric field equal to: 


$$
\begin{gathered}
|E|=\frac{I_{0} \omega}{2 \pi \sqrt{\sigma^{2}+\epsilon^{2} \omega^{2}} R}+\frac{I_{0} l \omega}{2 \pi \sqrt{\sigma^{2}+\epsilon^{2} \omega^{2}} R^{3}} \\
|E|=I_{0} \omega\left(\frac{\alpha}{R}+\frac{\beta}{R^{3}}\right)
\end{gathered}
$$

This strong electric field belongs to the transient state of the electrode-tissue circuit. However, by increasing the stimulation frequency and repeating this situation, it is possible to stimulate the nerve system and changing the state of ion channels.

It is clear that a larger induction field intensity causes a stronger stimulation, but the electrochemical detrimental effects of current controls the upper bound of intensity. Our data and other experimental data $[1,73-79,90]$ show the positive effects of increasing frequency for the reduction of the injected charge amplitude, achieving a proper stimulation of neurons. In addition, experimental evidence $[37,51,52]$ show that the type of the axon (neuron) could affect the stimulation threshold, as will be discussed in more detail in the following section.

\section{Onset response and stimulation intensity}

Experimental data indicate that the high-frequency stimulation, by both in blocking and stimulating neurons [36, 39, 48, 49, 57-61, 80], results in the "onset response", which is the transitory volley of activity produced in the nerve (Figure 2). There are two suggestions to reduce this undesirable phenomenon. First, via smoothing the stimulation current [36] which causes intensification of the destructive faradaic current [91]. The second one is the reduction of the stimulation intensity, e.g. by applying a lower amplitude field [36]. The drawback of decreasing the applied field amplitude is the reduction in the nerve response to stimulation. However, as mentioned above, by using a more square waveform shape stimulation and increasing its frequency, it is possible to overcome this problem and to achieve a weak but effective stimulation.

\section{Second Stage:}

In continuous ultra-high frequency stimulation, many neurons show a reduction in the firing frequency of their spike response, following an initial increase (Figure 2). This happens because of several phenomena, such as spike-frequency adaptation [36], fast neural adaptation, and synaptic fatigue $[37,40-43]$. 
This undesirable behavior can be reduced by stopping the stimulation and giving neurons enough time (recovery time) to return to their normal condition and then repeating the stimulation.

Recovery time depends on the inherent properties of stimulated neurons (equivalent RC time) and stimulation parameters, such as amplitude. This process, i.e. switching stimulation off and on periodically in the natural frequency of neurons, is the basic principle of the stimulation at ultrahigh frequencies.

Figure 6 which was adapted from the references [51,52], shows the dependency of recovery time to stimulation amplitudes. It is worthy to note that in the low amplitude stimulation, the recovery time is more a function of the size and type of excitable neurons [56].

\section{The effect of the shape and type of axon in high-frequency}

\section{stimulation}

The relation between axon diameter and the size of the effective surface of excitation, and the intrinsic electrical properties of the axon, highlights the role of axonal diameter in the ultra-high frequency stimulation in two ways:

1. The experimental results $[51,52]$ show that the recovery time is directly related to the minimum number of cycles in each stimulation period. Hence based on the length of recovery time, the length of the stimulation period is specified. This suggests that the recovery time determines the switching frequency.

2. It is well known that there is an inverse correlation between refractory period [this is the time during which another stimulus given to the neuron (no matter how strong) would not lead to a second action potential.] and axon's diameter. In fact, the distal absolute refractory period was inversely correlated with distal conduction velocity. As a result, the axon diameter is one of the parameters which controls the upper limit of the firing rate [56]. Thus, larger diameter could cover a larger range of firing frequency. Hence the larger the axon's diameter, the shorter the duration of the action potential and refractory period, and hence the larger the maximal frequency of firing $[92,93]$. The authors suggest a relationship between the axon diameter and the information rate so the axon diameter would be the main determinant of the properties of signal propagation along such axons. 
Hence, increasing the neuron diameter, along with rising the time of recovery and neuron rest, results in neuron firing at the lower cycle number of stimulation at a constant intensity. It also causes an increase in the frequency range capacity of the random frequency stimulation. Results from both the empirical data $[37,51,52]$ and the simulation [our unpublished simulation result], support the idea of stimulation in the lower cycles, by increasing of the neuron diameters in the pain, motor, and sensory neurons.

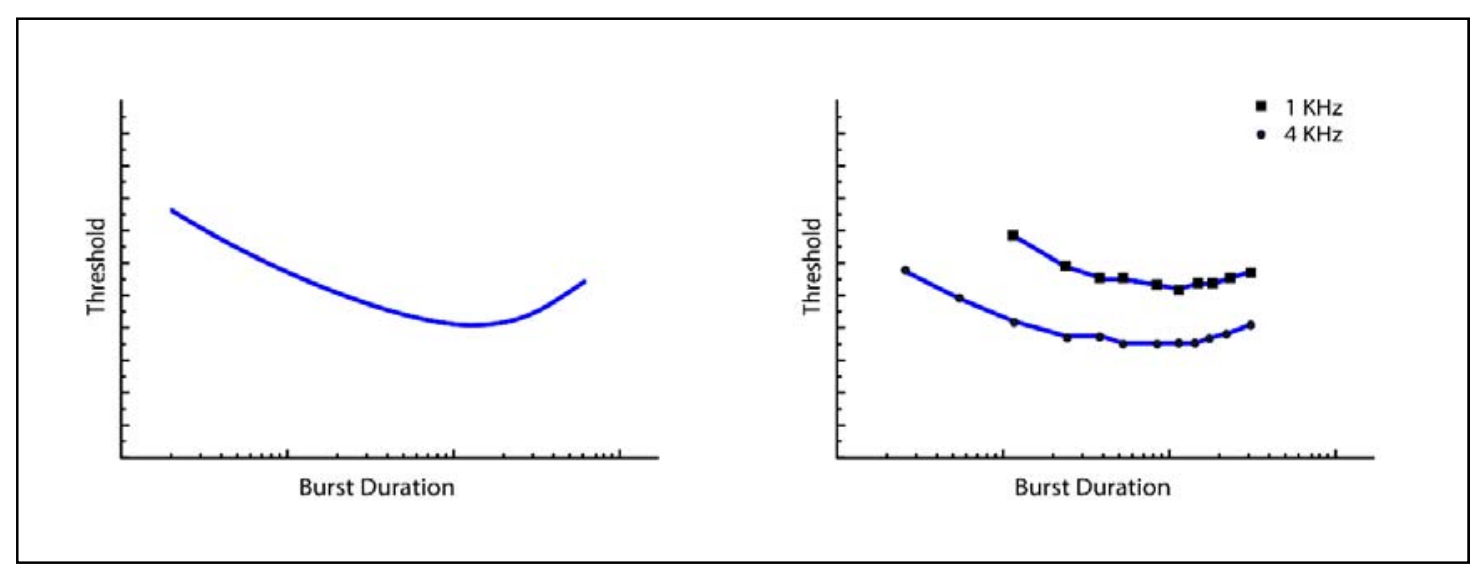

Figure 6. Diagrams indicate the relation between the threshold and minimum burst duration of stimulation that result in neuron firing. this figure is a schematic of the result of the references [51, 52].

this could be extended as a rule that: for thinner neurons, the stimulation threshold is increased [51]. In addition, due to different recovery time in the motor, sensory and pain neurons, the switching frequency is different and is a function of the neuron type [51]. Hence it seems that by tuning switching frequency, it is possible to optimize stimulation for a specific neuron type. However, our results still show a reduction in the central nervous system's response to the ultrahigh frequency stimulation, for which it is proportional to the frequency. This new type of reduction could be termed behavioral reduction or informatics reduction and is discussed in more detail in the following. 


\section{Neural informational adaptation}

Neural adaptation is a change over time in the responsiveness of the nerve system to a constant stimulus. By this phenomenon, even with an ultra-high frequency, modulated by a low-frequency carrier signal, the nerve system response decays gradually. Interestingly, our result shows that, like fatigue effect [37, 40-43], the rate of decay is proportional to the frequency in the central nerve system. Delivered information integrates and processes in the CNS. According to the authors' speculation, how CNS process analyzes and evaluates this information and what information is transmitted during stimulation could be inspiring in designing new experiments and new methods for getting more efficient stimulation. In principle, the symmetrical structure of the stimulus signal (which have a low entropy, and contain little information) causes a uniform and symmetrical stimulation of neurons. This uniformity of stimulation markedly reduces the time of CNS adaptation [As G. Ellis et all have mentioned in page 308 of his book titled 'How can physics underlie the mind?'[94]: “Animals learn to distinguish events that occur regularly together from those that are only randomly associated. Thus, the brain has evolved a mechanism that 'makes sense' out of events in the environment by assigning a predictive function to some events, based on past experience and learned rules of behavior [95]. This predictive capacity is built into the continually adapting connections in neural networks in the brain [96]. Each region of the cortex has a repertoire of sequences it knows, and it has a name for each sequence it knows[96] p. 129. And the known must be distinguished from what is new..."]. Hence, by variation of the stimulus signal, it is possible to increase the input information and reduce the adaptation rate. On the other word, for the same amplitude, this method could get a more efficient stimulus in respect of neural total respond and adaptation (especially in the sensory neurons which have wider effective frequency capacity [56]). Given that, random (informational) packets can carry more information [97]. Our suggestion for more effective stimulation is to create a high-frequency stimulation, modulated with a square-wave modulator, whose frequency is random, with Poisson distribution and neuron physiological frequency mean. (Figure 7)

Our result shows that at a high-frequency stimulus, which is switched on and off randomly according to a Poisson distribution, with mean $100 \mathrm{~Hz}$ and $10 \%$ duty, even a very low discharge in each period $(\sim 0.3 n c)$ results in a nerve stimulation and a macroscopic response of the subject. It is worth to note that with the same stimulation conditions, but with a uniform switching frequency, we didn't see any macroscopic response in the subject. 


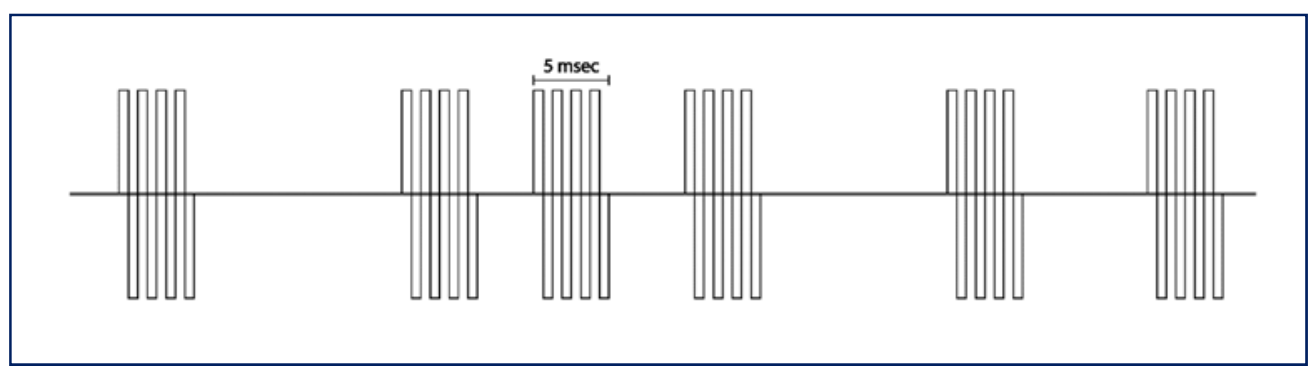

Figurev. A high-frequency stimulation, modulated with a square-wave modulator whose frequency is random with a Poisson distribution and a neuron physiological frequency mean.

In addition, due to the variety of axons and their frequency carrier capacity [56], we speculate that the stimulation with random high frequency, with regular/random switching on and off, can utilize the neuron frequency capacity more efficiently and is also suitable for thicker neurons, such as sensory or brain neurons due to their broad firing frequency coverage. We didn't perform any experiment around this idea, but in this context, may be the result of ref [98] could be justified. The rectangular signal stimulation of this research includes the random pulse in duration or amplitude. Since the Fourier transform of the rectangular pulse has a wide range of frequency proportional to the inverse of duration and considering its randomness, this method would be justified in the above-mentioned context (the random high frequency with regular switching) (Figure 8). Also, random stimulation amplitude results in random firing rate response of neurons.

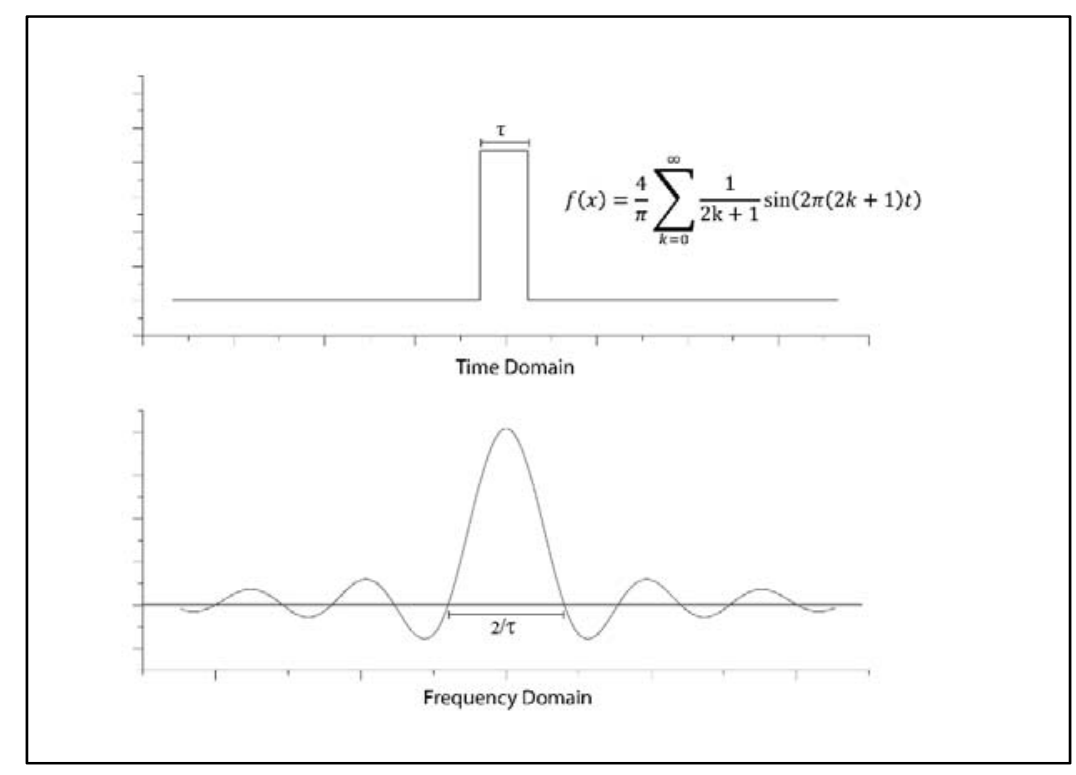

Figure 8. Fourier transform of a rectangular pulse. As the pulse duration is made shorter the signal frequency range becomes wider and it includes the higher frequency component. 


\section{Our data on random stimulation}

All experimental procedures were in accordance with the guidelines of the National Institutes of Health and the Iranian Society for Physiology and Pharmacology and were approved by the animal care and use committee of the Institute for Research in Fundamental Sciences.

1. We had the stimulation of clarias gariepinus motor neuron (control system) located in front of the cerebellum. As we reported in detail in [99], for testing the above hypothesis we stimulated the motor system of clarias gariepinus in front of its cerebellum. The stimulation was done by an implanted stainless steel electrode $(1 \cdots \mu \mathrm{m}$ diameter, and $1.0 \mathrm{~mm}$ depth). The range of stimulation signal was between $2.5 \mathrm{KHz}$ to $1 \mathrm{MHz}$, and the base signal (on and off switcher) is between $100 \mathrm{~Hz}$ to $200 \mathrm{~Hz}$, with the duty varied from $5 \%$ to $20 \%$. As expected, due to the capacitive properties of the ionic environment at the high frequency, the DC current, which passes through tissue, was almost zero (with $2 \%$ precision). In this experiment, the storage charge in each cycle at $200 \mathrm{KHz}$ was equal to $3 n C$.

Table 1 shows the minimum discharge in each stimulation when a significant macroscopic response was observed.

Table 1: The minimum discharge value in each fish brain stimulation, when a meaningful macroscopic response was observed.

\begin{tabular}{|c|c|c|c|c|}
\hline $\begin{array}{c}\text { Type of } \\
\text { stimulation }\end{array}$ & $\begin{array}{c}\text { On \& off Mean } \\
\text { frequency }(\mathrm{Hz})\end{array}$ & $\begin{array}{c}\text { Frequency of } \\
\text { stimulation }\end{array}$ & $\begin{array}{c}\text { Charge amplitude } \\
(\mathrm{nc})\end{array}$ \\
\hline Random & 200 & $1 \mathrm{MHz}$ & 5 & 0.3 \\
\hline Random & 200 & $200 \mathrm{KHz}$ & 5 & 3 \\
\hline Regulate & 200 & $3.5 \mathrm{KHz}$ & 5 & 30 \\
\hline Regulate & 150 & $150 \mathrm{~Hz}$ & 5 & 60 \\
\hline
\end{tabular}

As expected, we didn't see any macroscopic response in the direct (continues) ultra-high frequency stimulation. However, the fish motor system was stimulated and the fish swam forward when the stimulation switched on and off at the low frequency $(100 \mathrm{~Hz}-200 \mathrm{~Hz})$ 
2. Weak stimulation of afferent nerves.

The electrode is the same as in the previous experiment, but its depth was $2 \mathrm{~mm}$. Table 2 shows the amount of discharge in each period when a significant macroscopic response was observed. As was mentioned earlier in our model, we expect that at high frequencies the system rapidly adapts itself as was observed in our experiment [we observe that depending on the intensity of stimulation, the subject does not respond absolutely or after a few rotations, it stops the responses]. When the signal frequency is superimposed on a low-frequency carrier wave, we observed a meaningful slowdown in the adaptation. Furthermore, as the model predicts, by randomizing the carrier wave (with a Poisson distribution, with mean $200 \mathrm{~Hz}$ and $15 \%$ duty), the stimulation efficiency was greatly improved, and the motor response increased sharply. Interestingly, a low field intensity stimulation which was carried by a low constant frequency had, sometimes, no effect on the subject, and changing the constant frequency with a random one, caused a smooth response from the subject. movie 1 shows the respond of fish under later stimulation method.

Table 2: The amount of discharge in each period, when a significant macroscopic response was observed in the fish locomotion.

\begin{tabular}{|c|c|c|c|c|}
\hline $\begin{array}{c}\text { Type of } \\
\text { stimulation }\end{array}$ & $\begin{array}{c}\text { On \& off Mean } \\
\text { frequency }(\mathrm{Hz})\end{array}$ & $\begin{array}{c}\text { Frequency of } \\
\text { stimulation }\end{array}$ & Duty (\%) & Charge amplitude (nc) \\
\hline Random & 200 & $300 \mathrm{KHz}$ & 15 & 0.7 \\
\hline random & 200 & $2.5 \mathrm{KHz}$ & 15 & 12 \\
\hline regulate & 150 & $150 \mathrm{~Hz}$ & 15 & 140 \\
\hline
\end{tabular}

\section{Conclusion}

In recent years there has been increased attention to the ultra-high frequency electrical stimulation of the brain and nervous system. This type of stimulation, due to its less destructive effects, is suitable for medical and cyborg applications. In this article, we provide a brief description of the mechanism underlying the ultra-high frequency stimulation. Then, from an informatics perspective, we proposed a new signaling method for the low amplitude ultra-high frequency stimulation. In this method, we have tried to reduce adaptation of nerve system by modulating the stimulation signal via a low frequency rectangular random wave. By this method, we could reach the "almost zero discharge" with minimum destructive effect. Hence this method could be 
applicable to the human DBS and other medical electrical stimulation of the nervous system. As we mentioned, a weak intensity stimulation causes an increased probability of neuron firing at the natural environmental noises. We speculate that this phenomenon result in an asymmetric firing rate in the stimulated area compare to other regions however this description needs more investigations.

\section{Author Contributions}

M. J. and Y. J. designed the research, conceived the experiments, and developed the method and theory. The theory and data were analyzed and controlled by M. G. The results were discussed and interpreted by M. J. The manuscript was written and revised by M. G. M. J. and Y. J.

\section{Additional Information}

The authors declare that they have no competing interests.

\section{References}

1. Merrill, D.R., M. Bikson, and J.G. Jefferys, Electrical stimulation of excitable tissue: design of efficacious and safe protocols. J Neurosci Methods, 2005. 141(2): p. 171-98.

2. Ghovanloo, M. Switched-capacitor based implantable low-power wireless microstimulating systems. in Circuits and Systems, 2006. ISCAS 2006. Proceedings. 2006 IEEE International Symposium on. 2006. IEEE.

3. Wei, X.F., Analysis and design of electrodes for deep brain stimulation. 2009, Duke University.

4. Harnack, D., et al., The effects of electrode material, charge density and stimulation duration on the safety of high-frequency stimulation of the subthalamic nucleus in rats. $\mathrm{J}$ Neurosci Methods, 2004. 138(1-2): p. 207-16.

5. Guyton, D.L. and F.T. Hambrecht, Theory and design of capacitor electrodes for chronic stimulation. Med Biol Eng, 1974. 12(5): p. 613-20.

6. Ayers, J.L. and W.J. Davis, Neuronal control of locomotion in the lobster, Homarus americanus. Journal of Comparative Physiology A: Neuroethology, Sensory, Neural, and Behavioral Physiology, 1977. 115(**50cyborgi@12i): p. 1-27.

7. Cai, L., et al., Modulating Motor Behaviors by Electrical Stimulation of Specific Nuclei in Pigeons. Journal of Bionic Engineering, 2015. 12(4): p. 555-564. 
8. Kobayashi, N., et al., Artificial control of swimming in goldfish by brain stimulation: confirmation of the midbrain nuclei as the swimming center. Neuroscience letters, 2009. 452(1): p. 42-46.

9. Lin, J.-y., et al. Using dlPAG-evoked immobile behavior in animal-robotics navigation. in Computer Science and Education (ICCSE), 2010 5th International Conference on. 2010. IEEE.

10. Uematsu, K. and T. Todo, Identification of the midbrain locomotor nuclei and their descending pathways in the teleost carp, Cyprinus carpio. Brain research, 1997. 773(1-2): p. $1-7$.

11. Yang, J., et al., A robo-pigeon based on an innovative multi-mode telestimulation system. Biomed Mater Eng, 2015. 26 Suppl 1: p. S357-63.

12. Howell, B., L.E. Medina, and W.M. Grill, Effects of frequency-dependent membrane capacitance on neural excitability. J Neural Eng, 2015. 12(5): p. 056015-56015.

13. Li, P. and N.F. Uren, Analytical solution for the electric potential due to a point source in an arbitrarily anisotropic half-space. Journal of Engineering Mathematics, 1998. 33(2): p. 129-140.

14. Lee, W.H., et al., Regional electric field induced by electroconvulsive therapy in a realistic finite element head model: influence of white matter anisotropic conductivity. Neuroimage, 2012. 59(3): p. 2110-23.

15. Peterchev, A.V., et al., ECT stimulus parameters: rethinking dosage. The journal of ECT, 2010. 26(3): p. 159.

16. Hofmann, L., et al., Modified pulse shapes for effective neural stimulation. Front Neuroeng, 2011. 4: p. 9.

17. Wongsarnpigoon, A., J.P. Woock, and W.M. Grill, Efficiency analysis of waveform shape for electrical excitation of nerve fibers. IEEE Trans Neural Syst Rehabil Eng, 2010. 18(3): p. 319-28.

18. Altman, K.W. and R. Plonsey, Development of a model for point source electrical fibre bundle stimulation. Med Biol Eng Comput, 1988. 26(5): p. 466-75.

19. Clark, J. and R. Plonsey, A mathematical evaluation of the core conductor model. Biophys J, 1966. 6(1): p. 95-112.

20. Rall, W., Core conductor theory and cable properties of neurons, in Comprehensive physiology. 2011. p. 39-97.

21. Rattay, F., Analysis of models for extracellular fiber stimulation. IEEE Trans Biomed Eng, 1989. 36(7): p. 676-82.

22. Leung, R.T., et al., In vivo and in vitro comparison of the charge injection capacity of platinum macroelectrodes. IEEE Trans Biomed Eng, 2015. 62(3): p. 849-57.

23. Joucla, S., A. Gliere, and B. Yvert, Current approaches to model extracellular electrical neural microstimulation. Front Comput Neurosci, 2014. 8: p. 13.

24. Maharbiz, M.M. and H. Sato, Cyborg beetles. Sci Am, 2010. 303(6): p. 94-9.

25. Sato, H., et al. Radio-controlled cyborg beetles: a radio-frequency system for insect neural flight control. in Micro Electro Mechanical Systems, 2009. MEMS 2009. IEEE 22nd International Conference on. 2009. IEEE.

26. Bernau, N.A., R.L. Puzdrowski, and R.B. Leonard, Identification of the midbrain locomotor region and its relation to descending locomotor pathways in the Atlantic stingray, Dasyatis sabina. Brain Res, 1991. 557(1-2): p. 83-94.

27. Bozkurt, A., J. F. Gilmour R, and A. Lal, Balloon-assisted flight of radio-controlled insect biobots. IEEE Trans Biomed Eng, 2009. 56(9): p. 2304-7. 
28. Choo, H.Y., et al., Electrical Stimulation of Coleopteran Muscle for Initiating Flight. PLoS One, 2016. 11(4): p. e0151808.

29. Erickson, J.C., et al., Effective Stimulus Parameters for Directed Locomotion in Madagascar Hissing Cockroach Biobot. PLoS One, 2015. 10(8): p. e0134348.

30. Holzer, R. and I. Shimoyama. Locomotion control of a bio-robotic system via electric stimulation. in Intelligent Robots and Systems, 1997. IROS'97., Proceedings of the 1997 IEEE/RSJ International Conference on. 1997. IEEE.

31. Sanchez, C.J., et al., Locomotion control of hybrid cockroach robots. J R Soc Interface, 2015. 12(105): p. 20141363.

32. Sato, H., et al., Remote radio control of insect flight. Front Integr Neurosci, 2009. 3: p. 24.

33. Sato, H. and M.M. Maharbiz, Recent developments in the remote radio control of insect flight. Frontiers in Neuroscience, 2010. 4: p. 199.

34. Shik, M.L., F.V. Severin, and G.N. Orlovsky, Control of walking and running by means of electrical stimulation of the mesencephalon. Electroencephalogr Clin Neurophysiol, 1969. 26(5): p. 549.

35. Volkmann, J., E. Moro, and R. Pahwa, Basic algorithms for the programming of deep brain stimulation in Parkinson's disease. Movement Disorders, 2006. 21: p. S284-S289.

36. Kilgore, K.L. and N. Bhadra, Reversible nerve conduction block using kilohertz frequency alternating current. Neuromodulation: Technology at the Neural Interface, 2014. 17(3): p. 242-255.

37. Ward, A.R., Electrical stimulation using kilohertz-frequency alternating current. Physical therapy, 2009. 89(2): p. 181.

38. Cahana, A., et al., Pulsed radiofrequency: current clinical and biological literature available. Pain Med, 2006. 7(5): p. 411-23.

39. Woo, M.Y. and B. Campbell, Asynchronous Firing and Block of Peripheral Nerve Conduction by 20 Kc Alternating Current. Bull Los Angel Neuro Soc, 1964. 29: p. 87-94.

40. Jones, D.A., High-and low-frequency fatigue revisited. Acta Physiol Scand, 1996. 156(3): p. $265-70$.

41. Jones, D., Muscle fatigue due to changes beyond the neuromuscular junction. Human muscle fatigue: physiological mechanisms, 1981. 82: p. 178-196.

42. Sawan, M., et al., Stimulator design and subsequent stimulation parameter optimization for controlling micturition and reducing urethral resistance. IEEE Trans Rehabil Eng, 1996. 4(1): p. 39-46.

43. Solomonow, M., et al., Fatigue considerations of muscle contractile force during highfrequency stimulation. Am J Phys Med, 1983. 62(3): p. 117-22.

44. $\quad$ Cogan, S.F., Neural stimulation and recording electrodes. Annu Rev Biomed Eng, 2008. 10: p. 275-309.

45. Brummer, S.B., L.S. Robblee, and F.T. Hambrecht, Criteria for selecting electrodes for electrical stimulation: theoretical and practical considerations. Ann N Y Acad Sci, 1983. 405: p. 159-71.

46. Pudenz, R.H., et al., Electrical stimulation of the brain. II. Effects on the blood-brain barrier. Surg Neurol, 1975. 4(2): p. 265-70.

47. Kuncel, A.M. and W.M. Grill, Selection of stimulus parameters for deep brain stimulation. Clin Neurophysiol, 2004. 115(11): p. 2431-41.

48. Gildemeister, M., Zur theorie des elektrischen Reizes. V. Polarisation durch Wechselströme. Berichte über die Verhandlungen der Sächsischen Akademie der Wissenschaften zu Leipzig Mathematisch-Physische Klasse, 1930. 81: p. 303-313. 
49. Gildemeister, M., Untersuchungen über die Wirkung der Mittelfrequenzströme auf den Menschen. Pflügers Archiv European Journal of Physiology, 1944. 247(4): p. 366-404.

50. Furlanetti, L.L., et al., Continuous High-Frequency Stimulation of the Subthalamic Nucleus Improves Cell Survival and Functional Recovery Following Dopaminergic Cell Transplantation in Rodents. Neurorehabil Neural Repair, 2015. 29(10): p. 1001-12.

51. Ward, A.R. and S. Lucas-Toumbourou, Lowering of sensory, motor, and pain-tolerance thresholds with burst duration using kilohertz-frequency alternating current electric stimulation. Archives of physical medicine and rehabilitation, 2007. 88(8): p. 1036-1041.

52. Ward, A.R. and W.L.H. Chuen, Lowering of sensory, motor, and pain-tolerance thresholds with burst duration using kilohertz-frequency alternating current electric stimulation: part II. Archives of physical medicine and rehabilitation, 2009. 90(9): p. 1619-1627.

53. Bhadra, N., et al., Simulation of high-frequency sinusoidal electrical block of mammalian myelinated axons. J Comput Neurosci, 2007. 22(3): p. 313-26.

54. Tai, C., W.C. de Groat, and J.R. Roppolo, Simulation of nerve block by high-frequency sinusoidal electrical current based on the Hodgkin-Huxley model. IEEE Trans Neural Syst Rehabil Eng, 2005. 13(3): p. 415-22.

55. Wang, Y., et al., Visual cue-guided rat cyborg for automatic navigation [research frontier]. IEEE Computational Intelligence Magazine, 2015. 10(2): p. 42-52.

56. Perge, J.A., et al., Why do axons differ in caliber? Journal of Neuroscience, 2012. 32(2): p. 626-638.

57. Bhadra, N. and K.L. Kilgore, High-frequency electrical conduction block of mammalian peripheral motor nerve. Muscle Nerve, 2005. 32(6): p. 782-90.

58. Bowman, B.R. and D.R. McNeal, Response of single alpha motoneurons to high-frequency pulse trains. Stereotactic and Functional Neurosurgery, 1987. 49(3): p. 121-138.

59. Cuellar, J.M., et al., Effect of High-Frequency Alternating Current on Spinal Afferent Nociceptive Transmission. Neuromodulation: Technology at the Neural Interface, 2013. 16(4): p. 318-327.

60. Miles, J., et al., Effects of ramped amplitude waveforms on the onset response of highfrequency mammalian nerve block. Journal of neural engineering, 2007. 4(4): p. 390.

61. Tai, C., J.R. Roppolo, and W.C. de Groat, Block of external urethral sphincter contraction by high frequency electrical stimulation of pudendal nerve. J Urol, 2004. 172(5 Pt 1): p. 2069-72.

62. Nemec, H., Interferential therapy: a new approach in physical medicine. Br J Physiother, 1959. 12: p. 9-12.

63. Nemec, H., Reizstromtherapie mit interferenzströmen. Dtsch Badebetrieb, 1960. 51: p. 320-323.

64. Nemec, H., Endogene elektrostimulierung durch mittelfrequente und interferenzströme. Rehabilitation, 1967. 20: p. 1-3.

65. Nemec, H., Elektrostimulierung in endogener Anwendung. Aktionsmechanismus der Interferenztherapie. Physikalische Medizin und Rehabilitation, 1968. 9: p. 73-75.

66. Robertson, V.J., et al., Electrotherapy explained: principles and practice. 2006: Elsevier Health Sciences.

67. Watson, T., Electrotherapy: evidence-based practice. 2008: Elsevier Health Sciences.

68. Currier, D.P., R.M. Nelson, and K.W. Hayes, Clinical Electrotherapy. 1999: Appleton \& Lange.

69. Andrianova, G., et al., Primenenie elektrostimuliatsii dlia trenirovki mishechnoj sili. Novosti Meditsinskogo Priborostroeniia, 1971. 3(***11i): p. 40-47. 
70. Dantas, L.O., et al., Comparison between the effects of 4 different electrical stimulation current waveforms on isometric knee extension torque and perceived discomfort in healthy women. Muscle \& nerve, 2015. 51(1): p. 76-82.

71. Medina, L.E. and W.M. Grill, Nerve excitation using an amplitude-modulated signal with kilohertz-frequency carrier and non-zero offset. Journal of NeuroEngineering and Rehabilitation, 2016. 13(***12i): p. 63.

72. van Dongen, M.N., et al., High frequency switched-mode stimulation can evoke post synaptic responses in cerebellar principal neurons. Frontiers in neuroengineering, 2015. 8(2).

73. Lapicque, L., Quantitative investigations of electrical nerve excitation treated as polarization. 1907. Biological cybernetics, 2007. 97(5-6): p. 341.

74. McCreery, D.B., T.G. Yuen, and L.A. Bullara, Chronic microstimulation in the feline ventral cochlear nucleus: physiologic and histologic effects. Hear Res, 2000. 149(1-2): p. 223-38.

75. McCreery, D.B., et al., Accessing the tonotopic organization of the ventral cochlear nucleus by intranuclear microstimulation. IEEE Trans Rehabil Eng, 1998. 6(4): p. 391-9.

76. Moro, E., et al., The impact on Parkinson's disease of electrical parameter settings in STN stimulation. Neurology, 2002. 59(5): p. 706-13.

77. Rizzone, M., et al., Deep brain stimulation of the subthalamic nucleus in Parkinson's disease: effects of variation in stimulation parameters. J Neurol Neurosurg Psychiatry, 2001. 71(2): p. 215-9.

78. Su, P.C., H.M. Tseng, and H.M. Liu, Unsuccessful deep brain stimulation in the subthalamic nucleus for advanced Parkinson's disease. Mov Disord, 2003. 18(3): p. 3501.

79. Volkmann, J., et al., Introduction to the programming of deep brain stimulators. Mov Disord, 2002. 17 Suppl 3: p. S181-7.

80. Kilgore, K.L. and N. Bhadra, Nerve conduction block utilising high-frequency alternating current. Med Biol Eng Comput, 2004. 42(3): p. 394-406.

81. Williamson, R.P. and B.J. Andrews, Localized electrical nerve blocking. IEEE Trans Biomed Eng, 2005. 52(3): p. 362-70.

82. Boido, D., et al., Stimulus-evoked potentials contribute to map the epileptogenic zone during stereo-EEG presurgical monitoring. Hum Brain Mapp, 2014. 35(9): p. 4267-81.

83. Ostrowsky, K., et al., Representation of pain and somatic sensation in the human insula: a study of responses to direct electrical cortical stimulation. Cerebral Cortex, 2002. 12(4): p. 376-385.

84. van't Klooster, M.A., et al., Time-frequency analysis of single pulse electrical stimulation to assist delineation of epileptogenic cortex. Brain, 2011(Pt 10): p. awr211.

85. Joucla, S. and B. Yvert, Modeling extracellular electrical neural stimulation: from basic understanding to MEA-based applications. J Physiol Paris, 2012. 106(3-4): p. 146-58.

86. Wagenaar, D.A., J. Pine, and S.M. Potter, Effective parameters for stimulation of dissociated cultures using multi-electrode arrays. Journal of neuroscience methods, 2004. 138: p. 27-37.

87. Bossetti, C.A., M.J. Birdno, and W.M. Grill, Analysis of the quasi-static approximation for calculating potentials generated by neural stimulation. J Neural Eng, 2008. 5(1): p. 44-53.

88. Jack, J.J.B., D. Noble, and R.W. Tsien, Electric current flow in excitable cells. 1975: Clarendon Press Oxford. 
89. Plonsey, R. and D.B. Heppner, Considerations of quasi-stationarity in electrophysiological systems. Bull Math Biophys, 1967. 29(4): p. 657-64.

90. Bronzino, J.D., Biomedical engineering handbook. Vol. 2. 1999: CRC press.

91. Bard, A.J., et al., Electrochemical methods: fundamentals and applications. Vol. 2. 1980: Wiley New York.

92. Pajevic, S. and P.J. Basser, An optimum principle predicts the distribution of axon diameters in normal white matter. PLoS One, 2013. 8(1): p. e54095.

93. Perge, J.A., et al., How the optic nerve allocates space, energy capacity, and information. Journal of Neuroscience, 2009. 29(24): p. 7917-7928.

94. Ellis, G. and SpringerLink, How Can Physics Underlie the Mind? Top-Down Causation in the Human Context, in The Frontiers Collection,. 2016, Springer Berlin Heidelberg : Imprint: Springer,: Berlin, Heidelberg. p. XXVI, 482 p. 36 illus.

95. Kandel, E.R., Biology and the future of psychoanalysis: a new intellectual framework for psychiatry revisited. Am J Psychiatry, 1999. 156(4): p. 505-24.

96. Hawkins, J. and S. Blakeslee, On intelligence. 1st ed. 2004, New York: Times Books. 261 p.

97. Shannon, C.E., A mathematical theory of communication. ACM SIGMOBILE Mobile Computing and Communications Review, 2001. 5(1): p. 3-55.

98. Donos, C., et al., A comparative study of the effects of pulse parameters for intracranial direct electrical stimulation in epilepsy. Clin Neurophysiol, 2016. 127(1): p. 91-101.

99. Jamali, M., Y. Jamali, and M. Golshani, Theory of cyborg. prepared for submission, 2017. 